\title{
Indicadores de prescrição médica: uma revisão sistemática
}

\section{Prescription indicators: a systematic review}

Recebido em: 02/07/2014 Aceito em: 23/09/2014
Pedro Henrique Rodrigues de SOUZA ${ }^{1}$, Maria Auxiliadora Silva OLIVEIRA ${ }^{1,2}$

${ }^{1}$ Instituto Superior de Teologia Aplicada - Inta. Rua Coronel Antonio Rodrigues Magalhães, 359, Dom Expedito, Sobral, CE, Brasil. ${ }^{2}$ Universidade Federal do Ceará - UFC. Rua Mauriocélio Rocha Ponte, 100, Derby, Sobral, CE, Brasil. E-mail:ecobio@zipmail.com.br

\section{ABSTRACT}

Prescription indicators help to establish a profile of the therapeutic practice in a region or health care facility, by using data such as: average number of pharmaceuticals per prescription, percentage of drugs prescribed by generic name, percentage prescribed drugs enrolled at essential drugs list, percentage of antibiotics prescribed, percentage of prescribed injectables. Therefore, the objective was to review published papers that addressed prescription indicators. Authors found usually more than 02 pharmaceuticals per prescription. For drugs prescribed by generic name, figures were lower than expected. The results show that more than $70 \%$ of the prescribed drugs are at Brazilian essential drugs list. Most of the papers present a very high rate of antibiotics, above to maximum level recommended (20\%). About injectable pharmaceutical dosage form, the results were favorable, with the exception of 01 author who found values above $10 \%$.

Keywords: prescription; prescription indicators; therapeutic profile

\section{RESUMO}

O farmacêutico, por meio da atenção farmacêutica, pode acompanhar a farmacoterapia, orientando e intervindo quando necessário, buscando melhoria na educação em saúde e na qualidade de vida do paciente. O objetivo deste trabalho foi realizar uma revisão bibliográfica sobre a importância da atenção farmacêutica na identificação e resolução das interações fármaco-nutriente, evidenciando as implicações dessas interações no efeito terapêutico do fármaco ou no estado nutricional. Foram realizadas pesquisas em livros e artigos científicos em bases de dados como Science Direct, Pubmed e Scielo, no período de 2000 a 2014. Os resultados mostraram que existem inúmeras possibilidades de interações fármaco-nutriente, as quais podem causar prejuízos ao efeito do fármaco e/ou ao estado nutricional do paciente, e essas muitas vezes não são identificadas, por falta de conhecimento dos profissionais da saúde ou devido aos testes de biodisponibilidade dos medicamentos serem realizados em jejum, por exigência dos órgãos sanitários. Desse modo, vê-se a necessidade da atuação do farmacêutico, que por meio do seu amplo conhecimento, pode colaborar com a diminuição dos riscos dessas interações fármaco-nutriente e nutriente-fármaco, resgatando assim a confiança do paciente e a essência da profissão.

Palavras Chave: atenção farmacêutica; interações alimento-fármaco; assistência ao paciente. 


\section{INTRODUÇÃO}

Desde 1993, a Organização Mundial de Saúde (OMS) vem insinuando a construção de indicadores selecionados de utilização de medicamentos buscando estratégias que possibilitem garantir uma farmacoterapia de qualidade, maior eficiência no emprego dos recursos disponíveis e a resolutividade dos serviços ofertados a partir de três níveis de diagnóstico: práticas de prescrição dos profissionais de saúde; assistência ao paciente e disponibilidade de fatores específicos de serviços propiciadores do uso racional, como existência de medicamentos essenciais e um mínimo de informação farmacêutica (1).

De acordo Farias et al. (2007) os indicadores de prescrição são úteis para descrever e avaliar aspectos que influenciam diretamente no Uso Racional de Medicamentos (URM) (2). Os indicadores baseiam-se nas práticas observadas em uma amostra de consultas realizadas nos serviços ambulatoriais de saúde para o tratamento de doenças agudas ou crônicas e permitem conhecer as práticas terapêuticas correntes, comparar parâmetros entre instituições similares e descrever as necessidades de medicamentos da população atendida, também reflete o funcionamento da assistência sanitária relacionada a vários aspectos essenciais do uso apropriado de medicamentos (1).

Diante da crescente preocupação em promover o URM, a OMS desenvolveu os Indicadores do Uso de Medicamentos como uma maneira de descrever e avaliar, com segurança, aspectos que afetam a prática farmacêutica em grandes e pequenos centros de saúde (1). Tais indicadores permitem conhecer as práticas terapêuticas correntes, comparar parâmetros entre instituições similares, descrever as necessidades de medicamentos da população atendida (3) e traçar um perfil da prática terapêutica de uma região, ou de uma unidade de saúde, usando alguns elementos de investigação tais como:

Número médio de medicamentos por prescrição: Oobjetivo desse indicador é medir o grau de polimedicação do paciente, visto ser este um dos fatores de interações medicamentosas e reações adversas. Permite observar a educação e a informação do prescritor (1).

Segundo Cosendey (2000) o padrão de prescrição de mais de 02 (dois) medicamentos por consulta determinaria a ocorrência da polimedicação. E a média brasileira para esse indicador (média de medicamento por receita) está acima do esperado (até 2,0) (4).

Outro estudo mostrou que se o número de medicamentos prescritos por paciente for maior que cinco, há maior risco de eventos adversos relacionados aos medicamentos. A idade da população deve ser levada em conta, uma vez que a tendência é aumentar o número de medicamentos por prescrição quanto mais idosa for a população (5). Contudo, um estudo realizado em 12 países pela OMS, a média de medicamentos prescritos variou de 1,3 a 3,8 por consulta (6).

Porcentagem de medicamentos prescritos pelo nome genérico: a prescrição pelo nome genérico do fármaco propicia o controle dos custos relacionados ao medicamento no serviço de saúde, devido ao fato de os medicamentos de referência geralmente serem mais onerosos $(1,2,7)$. Assim, de acordo com a OMS (1993), a finalidade desse indicador é determinar a tendência de se prescrever medicamentos pelo nome genérico, tendo como meta $100 \%$ dos medicamentos prescritos (1).

A Lei $n^{\circ} .9787$ de 10/02/1999, conhecida com a Lei dos Genéricos (8) obriga a denominação genérica na rede pública, pois isso facilita a educação, a informação, aumenta o acesso da população ao medicamento e promove a sua utilização racional $(9,10)$. A denominação genérica, além de ser de uso obrigatório por lei no serviço público brasileiro de saúde, também reduz as confusões entre nomes de medicamentos e facilita a obtenção de informações a respeito de fármacos em literatura internacional, pois a denominação comercial varia de país para país (11).

Porcentagem de medicamentos prescritos da lista de medicamentos essenciais: além de permitir controlar custos, a relação de medicamentos essenciais (RENAME) tem por objetivo garantir o tratamento para as principais patologias da população. Dessa forma, este indicador possibilita verificar o grau de adequação das prescrições à padronização de medicamentos (1).

Os medicamentos essenciais surgiram como meio de possibilitar o acesso aos medicamentos a um maior número de pessoas e sua adoção se baseia na segurança, eficácia terapêutica comprovada, qualidade e disponibilidade. Essa iniciativa alcançou adesão maior que a esperada. Então, a importância de se verificar o grau de conformidade do local com a política nacional de medicamentos, de modo essencial a se avaliar se a RENAME vai ao encontro da necessidade da população (12).

A seleção de medicamentos essenciais para os sistemas locais além de atender às necessidades, serve de suporte para decisões políticas, devendo ser um processo dinâmico com a participação dos profissionais de saúde. Oferece vantagens como: redução do número de produtos farmacêuticos a serem adquiridos, analisados, armazenados e distribuídos; melhoramento do emprego, 
gestão, informação e vigilância de medicamentos e estímulo às indústrias locais (13).

A questão dos medicamentos essenciais foi incorporada pela OMS durante a década de 70. Durante a $28^{\text {a }}$ Assembléia Mundial de Saúde, em 1975, foram revistos os principais problemas enfrentados pelos países em desenvolvimento no que se refere aos medicamentos e propostas novas diretrizes (14). A partir de uma avaliação da experiência adquirida nos países que haviam adotado esquemas de medicamentos essenciais, foi mostrado que a seleção destes medicamentos essenciais deveria ser realizada nos níveis locais e atualizada periodicamente com assessoria da comunidade científica. Foi dada ênfase à importância de promover informação adequada sobre as propriedades, indicações e utilização dos medicamentos selecionados (15). Estes medicamentos essenciais devem estar disponíveis para sociedade que deles necessitem, nas formas farmacêuticas apropriadas. Além disso, compõem uma relação nacional de referência que serve de base para o direcionamento da produção farmacêutica, para o desenvolvimento científico e tecnológico, e também para a definição de listas de medicamentos essenciais nos âmbitos estaduais e municipais, segundo a situação epidemiológica respectiva (8).

Segundo Carmo et al. (2003) se existe uma lista de medicamentos padronizados em um município, esta deve ser constituída pelos medicamentos essenciais para aquela localidade e por isso, deve ser seguida (10).

Além do não conhecimento da lista pelos prescritores, outros fatores que influenciam este indicador são a disponibilidade e efetiva divulgação desta lista nos serviços de saúde. Segundo Farias et al. (2007), em estudos realizados na cidade de Campina Grande (PB), por exemplo, o alto valor para este indicador $(91,90 \%)$ é atribuído à entrega da Relação Municipal de Medicamentos Essenciais (REMUME) a todas as unidades de saúde (2). Nos países em desenvolvimento, o uso da lista de medicamentos essenciais é muito importante, pois apresenta muitas vantagens em termos de economia e efetividade (4).

Porcentagem de antibióticos prescritos: 0 principal problema relacionado à prescrição excessiva e inapropriada de antibióticos é o desenvolvimento de resistência bacteriana, que pode acarretar graves consequências ao paciente (1).

Para esse indicador de prescrição, segundo Cosendey (2000) a porcentagem de consultas em que se prescreve um antibiótico deve ser $20 \%$ ou menos (4). Em média, $75 \%$ desses fármacos são prescritos inadequadamente e $50 \%$ os usuários os utilizam maneira inadequada (16). Consequentemente, avaliar o consumo de antibióticos pela população torna-se cada vez mais importante, pois há vários problemas relacionados ao seu uso incorreto e abusivo (2), dos quais o principal é o desenvolvimento de bactérias resistentes que, faz com que haja necessidade da administração de antibióticos mais potentes e, provavelmente, mais onerosos no tratamento de infecções causadas pelos mesmos microrganismos (7).

Porcentagem de injetáveis prescritos: apesar de importantes em diversas situações, a administração de injetáveis pode trazer sérias consequências quando erroneamente prescritos ou aplicados (9). A prescrição contendo pelo menos um medicamento injetável mede o seu grau de utilização. Com este parâmetro pode-se perceber a cultura de prescritores e pacientes no que diz respeito a esta forma farmacêutica (17). É preconizado para este indicador valores abaixo de $10 \%$ (4).

Medicamentos injetáveis são essenciais em algumas situações, porém seu abuso desperdiça recursos escassos e expõe pacientes a riscos de reações adversas e doenças (17). Santos e Nitrini (2004), citam como consequências do uso indevido dos injetáveis a sua aplicação incorreta, reações anafiláticas, necroses teciduais ou infecções por deficiência de assepsia (17). Ainda de acordo com esses autores, em estudos realizados pelos mesmos, encontraram injetáveis em $8,3 \%$ das prescrições no município de Ribeirão Preto (SP).

Considerando a importância dos indicadores para a saúde pública, o objetivo do presente trabalho foi fazer um levantamento de artigos que tratem da temática indicadores de prescrição médica e a partir dos resultados, traçar o perfil da prática terapêutica em Unidades Básicas de Saúde do território brasileiro.

\section{MÉTODO}

O presente estudo teve como base uma revisão da literatura sobre os indicadores de prescrições médicas e erros de prescrições englobando aspectos legais, tipos de indicadores, em trabalhos realizados em diversas cidades do Brasil.

Deste modo, foram selecionados artigos científicos que abordassem conceitos sobre indicadores de prescrições, a partir de busca em bancos de dados virtuais tais como: LILACS, BIREME, Scientific Library on Line (SCIELO), Google, além de documentos, artigos e dados publicados pelo Ministério da Saúde e Agência Nacional de Vigilância Sanitária (Anvisa).

A busca foi feita utilizando os seguintes descritores: prescrições; indicadores de prescrição; análise de prescrições. 
O critério de inclusão foi ser publicado no período compreendido entre 1986 e 2013. Para os indicadores de prescrição médica foram incluídos somente os artigos oriundos de pesquisas em Unidades Básicas de Saúde.

\section{RESULTADOS E DISCUSSÃO}

Número médio de medicamentos por prescrição: Esse indicador avalia o padrão de consumo de medicamento e o número de medicamentos prescrito, ou seja, o grau de polimedicação, permitindo-se detectar o sobre o uso de medicamento.
Foi possível observar, a partir da Tabela 1 que os municípios de Américo Brasiliense/SP (6) e Bagé/RS (24) possuíam o maior número médio de medicamentos por prescrição (média de 2,9) . Os autores, em seus artigos, comentaram que tal número elevado pode ser considerado um risco para interações medicamentosas e incompreensão da prescrição. A cidade de Piracicaba/SP (10) obteve o menor valor (média de 1,1). Interessante relembrar que ambos os trabalhos foram focados diretamente em pesquisa nas UBS nos municípios brasileiros.

Tabela 1: Número médio de medicamentos por prescrição observado nos serviços de saúde de municípios brasileiros (1986-2013)

\begin{tabular}{|c|c|c|c|}
\hline Cidade (Estado) & $\begin{array}{l}\text { Média de medicamentos } \\
\text { por prescrição }\end{array}$ & Referência) & \\
\hline Américo Brasiliense (SP) & 2,9 & Carneiro et al. (2000) & 6 \\
\hline Ponta Grossa (MT) & 2,6 & Marcondes (2002) & 19 \\
\hline Campo Grande (MS) & 2,3 & Cunha et al. (2002) & 11 \\
\hline Piracicaba (SP) & 1,1 & Carmo et al. (2003) & 10 \\
\hline Blumenau (SC) & 1,8 & Colombo et al. (2004) & 7 \\
\hline Belo Horizonte (MG) & 2,4 & Acurcio et al. (2004) & 3 \\
\hline Ribeirão Preto (SP) & 2,2 & Santos e Nitrini (2004) & 17 \\
\hline Brasília (DF) & 2,3 & Naves e Silver (2005) & 20 \\
\hline Ibiporã (PR) & 2,0 & Girotto e Silva (2006) & 9 \\
\hline Campinas Grande (PB) & 1,5 & Farias et al. (2007) & 2 \\
\hline Esperança (PB) & 2,4 & Portela et al. (2007) & 21 \\
\hline Patos de Minas (MG) & 2,0 & Felício (2007) & 22 \\
\hline Marília (SP) & 2,1 & Oliveira et al. (2009) & 23 \\
\hline Bagé (RS) & 2,9 & Menezes et al. (2009) & 24 \\
\hline Anápolis (G0) & 2,4 & Borges e Silva (2010) & 25 \\
\hline Santa Cruz do Sul (RS) & 1.8 & Frohlich e Mengue (2011) & 26 \\
\hline Lorena (SP) & 1,5 & Fleith et al. (2011) & 27 \\
\hline Amurel (SC) & 2,4 & Souza et al. (2012) & 28 \\
\hline
\end{tabular}

Entre os fatores que podem contribuir para o aumento deste indicador está a falta de eficiência e eficácia que estes profissionais neles depositam (19). De acordo com Santos e Nitrini (2004) existem algumas diferenças quanto às características do sistema de saúde, perfil socioeconômico e de morbimortalidade da população que impossibilitam inferências quanto aos diferentes resultados encontrados (17).

Segundo Oliveira et al. (2009), embora a OMS estabeleça um padrão para esse indicador, este não diferencia as faixas etárias, o que pode torná-lo insuficiente para comparação dos dados, como no caso da população idosa, que é aquela que mais utiliza os serviços de saúde e medicamentos (23).

Apesar das limitações que esse este indicador possa ter, conhecer o número médio de medicamento por consulta é de grande importância nos estudos de utilização de medicamentos, pois a polifarmácia favorece o sinergismo e o antagonismo não desejado, o descumprimento das prescrições de medicamentos não essenciais e gastos excedentes com aqueles de uso supérfluo $(21,23)$. Pesquisas mostram que se o número de medicamentos 
for maior que cinco, há maior risco de eventos adversos relacionados aos medicamentos $(5,26)$.

Porcentagem de medicamento prescritos pelo nome genérico: o uso do nome genérico nas prescrições oferece dois benefícios fundamentais: possibilita a todos os envolvidos, tanto profissionais da saúde como pacientes, informações que não se limitam ao material da propaganda; e possibilita ao usuário identificar os produtos, fomentando a concorrência, resultando em queda dos preços (29).

Tabela 2: Porcentagem de medicamentos prescritos pelo nome do

fármaco em serviços de saúde de municípios brasileiros (1986-2013)

\begin{tabular}{|l|c|l|c|}
\hline \multicolumn{1}{|c|}{ Cidade (Estado) } & $\begin{array}{c}\text { Medicamentos prescritos por } \\
\text { nome do fármaco (\%) }\end{array}$ & \multicolumn{2}{c|}{ Referência } \\
\hline Américo Brasiliense (SP) & 33,9 & Carneiro et al. (2000) & 6 \\
\hline Ponta Grossa (MT) & 71,0 & Marcondes (2002) & 19 \\
\hline Campo Grande (MS) & 84,3 & Cunha et al. (2002) & 11 \\
\hline Piracicaba (SP) & 56,0 & Carmo et al. (2003) & 10 \\
\hline Belo Horizonte (MG) & 80,2 & Acurcio et al. (2004) & 3 \\
\hline Ribeirão Preto (SP) & 30,6 & Santos e Nitrini (2004) & 17 \\
\hline Brasília (DF) & 73,2 & Naves e Silver (2005) & 20 \\
\hline Ibiporã (PR) & 70,2 & Girotto e Silva (2006) & 9 \\
\hline Campinas Grande (PB) & 84,2 & Farias et al. (2007) & 2 \\
\hline Esperança (PB) & 74,6 & Portela et al. (2007) & 21 \\
\hline Patos de Minas (MG) & Felício (2007) & 22 \\
\hline Marília (SP) & 65,5 & Oliveira et al. (2009) & 23 \\
\hline Bagé (RS) & 88,8 & Menezes et al. (2009) & 24 \\
\hline Santa Cruz do Sul (RS) & 81,8 & Frohlich e Mengue (2011) & 26 \\
\hline Amurel (SC) & 84,0 & Souza et al. (2012) & 28 \\
\hline
\end{tabular}

A Tabela 2 mostra que o município de Ribeirão Preto (SP) foi o que apresentou a menor média de prescrições contendo o nome do fármaco (30,6\%). Isso pode ser explicado porque a análise das prescrições compreendeu receitas do ano de 1998, antes da promulgação da Lei $n^{\circ}$. 9787 de 10\02\1999, conhecida como a Lei dos Genéri$\cos (8)$ que obriga a denominação genérica nas prescrições de medicamentos na rede pública. Seis e nove anos após a vigência da Lei foram feitas novas análises de prescrições e novamente foram obtidos valores baixos, por exemplo, no município de Ribeirão Preto (SP) com $30,6 \%$ (17) e Patos de Minas (MG) com 65,5\%, mostram que essa Lei ainda é pouca aplicada (22).

Após 10 a 13 anos da vigência da Lei dos Genéricos, foram feitas novas análises de prescrições e dessa vez os resultados mostraram um bom desempenho, por exemplo, no município de Marília (SP), que apresentou $88,8 \%$ (23) e Amurel/SC (28), 86,8\%. A Lei está sendo cada vez aplicada. A OMS recomenda a prescrição de medicamentos por meio da denominação genérica, pois isso facilita a educação, a informação, aumenta o acesso da população ao medicamento e promove a sua utilização racional (30).
Santos e Nitrini (2004) encontraram diferentes valores para este indicador, o que pode refletir um perfil diferenciado de padrão de comportamento dos prescritores nas diferentes regiões (17). Contudo, existem outros fatores que podem influenciar no comportamento dos prescritores nas diferentes regiões: indústrias farmacêuticas ao realizarem um forte marketing para o incremento do consumo; e a disponibilidade de várias apresentações medicamentosas para o mesmo princípio ativo (2, 16), fazendo com que o médico tenha dificuldade para prescrever pelo nome genérico.

\section{Porcentagem de medicamento prescrito da lista} de medicamento essenciais: são considerados medicamentos essenciais aqueles que atendem as necessidades da atenção à saúde de maior parte da população; portanto devem estar sempre disponíveis nas quantidades e formas farmacêuticas adequadas (31).

Dos dados obtidos (Tabela 3), somente 03 trabalhos mostraram valores abaixo do valor de referência, sendo que um deles, referente no município de Patos de Minas (MG), com $68,2 \%$, estava bem próximo ao valor esperado (22). Os outros dois trabalhos, mostraram 
valores muito baixo: município de Américo Brasiliense/SP (6) com 54,1\% e o município de Ibiporã/PR (9), com $58,4 \%$. Esses valores abaixo do valor de referência podem ser devidos a uma possível não adesão dos prescritores ou não conhecimento da lista de medicamentos essenciais.

Tabela 3: Medicamentos prescritos, em serviços de saúde de municípios brasileiros, com base na lista de medicamentos essenciais (1986-2013)

\begin{tabular}{|c|c|c|c|}
\hline Cidade (Estado) & $\begin{array}{l}\text { Medicamentos prescritos presentes } \\
\text { na lista de medicamentos essenciais }\end{array}$ & Referências & \\
\hline Américo Brasiliense (SP) & 54,1 & Carneiro et al. (2000) & 6 \\
\hline Ponta Grossa (MT) & 87,0 & Marcondes (2002) & 19 \\
\hline Campo Grande (MS) & 92,7 & Cunha et al. (2002) & 11 \\
\hline Piracicaba (SP) & 70,0 & Carmo et al. (2003) & 10 \\
\hline Blumenau (SC) & 82,0 & Colombo et al. (2004) & 7 \\
\hline Ribeirão Preto (SP) & 83,4 & Santos e Nitrini (2004) & 17 \\
\hline Brasília (DF) & 85,3 & Naves e Silver (2005) & 20 \\
\hline Ibiporã (PR) & 58,4 & Girotto e Silva (2006) & 9 \\
\hline Campinas Grande (PB) & 91,9 & Farias et al. (2007) & 2 \\
\hline Esperança (PB) & 78,3 & Portela et al. (2007) & 21 \\
\hline Patos de Minas (MG) & 68,2 & Felício (2007) & 22 \\
\hline Marília (SP) & 83,6 & Oliveira et al. (2009) & 23 \\
\hline Anápolis (G0) & 81,7 & Borges e Silva (2010) & 25 \\
\hline Santa Cruz do Sul (RS) & 81,0 & Frohlich e Mengue (2011) & 26 \\
\hline Amurel (SC) & 80,9 & Souza et al. (2012) & 28 \\
\hline
\end{tabular}

Além do desconhecimento da existência da lista, existem vários outros fatores que podem contribuir a esse indicador: a disponibilidade e a efetiva divulgação desta lista nos serviços de saúde. Segundo Farias et al. (2007), tais fatores contribuem o alto valor para esse indicador $(91,9 \%)$ no município de Campinas Grande (PB) com à entrega da REMUME a todas as unidades de saúde (2).

De acordo com Cunha et al. (2002), no município de Campo Grande (MS) a média foi alta em relação aos estudos revisados (11), como pode ser constatado na Tabela 3. Os autores consideraram que as oscilações observadas nos diferentes momentos da pesquisa, provavelmente, são devidas a problemas de abastecimento.

Este indicador demonstra quanto o município está em consonância com a Política Nacional de Medicamentos, e por isso, todo o esforço deve ser feito para este indicador ter valores cada vez mais altos.

Porcentagem de antibióticos prescritos por consulta: Esse indicador tem a finalidade de controlar o uso de antibióticos e evitar resistências bacterianas. A Tabela 4 contempla os dados encontrados na revisão.

Quanto a consultas nas quais foi prescrito ao menos um antibiótico, o município que obteve o maior valor foi Bagé (RS) com 41,8\% (24) e o menor foi o município de Piracicaba (SP) com 3\% (10). De acordo com Menezes et al. (2009), há evidências de que a especialidade médica que mais prescreve antimicrobianos é a pediatria (24).

Dos dados obtidos, somente 06 estiveram abaixo do valor de referência $(7,10,21,23,26,28)$ e o restante apresentou valores muito altos $(2,6,9,11,17,19,20$, $22,24,25)$. Esses valores abaixo do valor de referência constituem um ótimo resultado, pois o consumo de antibióticos pela população torna-se cada vez mais importante. Nos valores acima do valor de referência vários fatores podem ter influenciado nos resultados, por exemplo, a época do ano em que foram coletadas as informações, pois é comum um maior consumo de antibióticos no inverno do que no verão.

Segundo Marcondes (2002), é importante a averiguação de outros determinantes como a capacidade de diagnóstico e eleição da terapia pelos prescritores, crenças e expectativas dos pacientes em relação ao antibiótico e adequação da escolha dos antibióticos pelo colegiado que elabora a relação municipal de medicamentos (19). Há também a confiabilidade e acesso aos laboratórios de diagnóstico diferencial e exames radiográficos. 
Tabela 4: Antibióticos prescritos por consulta nos serviços de saúde de municípios brasileiros (1986-2013)

\begin{tabular}{|l|c|l|c|}
\hline \multicolumn{1}{|c|}{ Cidade (Estado) } & Prescrição de antibióticos (\%) & \multicolumn{2}{|c|}{ Referências } \\
\hline Américo Brasiliense (SP) & 31,3 & Carneiro et al. (2000) & 6 \\
\hline Ponta Grossa (MT) & 33,0 & Marcondes (2002) & 19 \\
\hline Campo Grande (MS) & 27,4 & Cunha et al. (2002) & 11 \\
\hline Piracicaba (SP) & 3,0 & Carmo et al. (2003) & 10 \\
\hline Blumenau (SC) & 12,5 & Colombo et al. (2004) & 7 \\
\hline Ribeirão Preto (SP) & 21,3 & Santos e Nitrini (2004) & 17 \\
\hline Brasília (DF) & 26,4 & Naves e Silver (2005) & 20 \\
\hline Ibiporã (PR) & 22,0 & Girotto e Silva (2006) & 9 \\
\hline Campinas Grande (PB) & 21,1 & Farias et al. (2007) & 2 \\
\hline Esperança (PB) & 9,4 & Portela et al. (2007) & 21 \\
\hline Patos de Minas (MG) & 28,1 & Felício (2007) & 22 \\
\hline Marília (SP) & 6,5 & Oliveira et al. (2009) & 23 \\
\hline Bagé (RS) & 41,8 & Menezes et al. (2009) & 24 \\
\hline Anápolis (G0) & 27,0 & Borges e Silva (2010) & 25 \\
\hline Santa Cruz do Sul (RS) & 8,0 & Frohlich e Mengue (2011) & 26 \\
\hline Amurel (SC) & 19,0 & Souza et al. (2012) & 28 \\
\hline
\end{tabular}

\section{Porcentagem de injetáveis prescritos por} consulta: Esse indicador reflete a preocupação de verificar as consequências que injetáveis podem causar quando prescritos ou aplicados de forma inadequada. $\mathrm{Na}$ Tabela 5 são apresentados os dados encontrados.

Dos estudos analisados, os municípios de Piracicaba/SP (10), Campina Grande/PB (2) e Anápolis/GO (25), apresentaram os menores valores de porcentagem sobre o uso de injetáveis $(1 ; 1,1$ e 1, respectivamente). Segundo Farias et al. (2007), uma das razões para o baixo valor encontrado para este indicador foi o fato de o estudo ter sido feito em uma época quente do ano, quando a população é menos exposta a doenças (2). Somente os estudos realizados nos municípios de Ponta Grossa (MT) e Campo Grande (MS), mostraram os maiores valores de porcentagem de prescrições de injetáveis (13\% e 10,2\%, respectivamente), ou seja, o valor preconizado para este indicador é abaixo que $10 \%$ (13).
Marcondes (2002) ressaltou que o clima da cidade contribui para o aumento dos casos de pneumonia e outras infecções respiratórias, aumentando também o uso de antibióticos e anti-inflamatórios. Entretanto, relatou que no momento da pesquisa havia opções de tratamentos orais em estoques nas UBS, com exceção de amoxicilina suspensão (19).

Medicamentos injetáveis são essenciais em alguns casos, porém seu abuso desperdiça recursos escassos e expõem pacientes a riscos de reações adversas e doenças (18). Giroto e Silva (2006) consideraram que o resultado encontrado no estudo pode ter sido influenciado pelas preferências de alguns prescritores pela via parenteral (9)

Frohlich e Mengue (2011) citaram dois fatores importantes que podem indicar o motivo de valores baixos para esse indicador: a lista de medicamentos essenciais dos municípios pode restringir esta forma farmacêutica, além das UBS só disponibilizarem injetáveis para procedimentos no próprio posto (26). 
Tabela 5: Medicamentos injetáveis prescritos, em serviços de saúde de municípios brasileiros, por consulta (1986-2013)

\begin{tabular}{|l|c|l|c|}
\hline \multicolumn{1}{|c|}{ Cidade (Estado) } & Prescrição de injetáveis por consulta (\%) & \multicolumn{2}{|c|}{ Referências } \\
\hline Américo Brasiliense (SP) & 9,9 & Carneiro et al. (2000) & 6 \\
\hline Ponta Grossa (MT) & 13,0 & Marcondes (2002) & 19 \\
\hline Campo Grande (MS) & 10,2 & Cunha et al. (2002) & 11 \\
\hline Piracicaba (SP) & 1,0 & Carmo et al. (2003) & 10 \\
\hline Blumenau (SC) & 8,1 & Colombo et al. (2004) & 7 \\
\hline Ribeirão Preto (SP) & 8,3 & Santos e Nitrini (2004) & 17 \\
\hline Brasília (DF) & 7.5 & Naves e Silver (2005) & 20 \\
\hline Ibiporã (PR) & 7,0 & Girotto e Silva (2006) & 9 \\
\hline Campinas Grande (PB) & 1,1 & Farias et al. (2007) & 2 \\
\hline Esperança (PB) & 2,9 & Portela et al. (2007) & 21 \\
\hline Patos de Minas (MG) & 4,8 & Felício (2007) & 22 \\
\hline Marília (SP) & 2,2 & Oliveira et al. (2009) & 23 \\
\hline Anápolis (G0) & Borges e Silva (2010) & 25 \\
\hline Santa Cruz do Sul (RS) & 1,0 & Frohlich e Mengue (2011) & 26 \\
\hline Amurel (SC) & 3,0 & Souza et al. (2012) & 28 \\
\hline
\end{tabular}

\section{CONCLUSÃO}

A revisão bibliográfica a partir dos indicadores da OMS pode ajudar a melhor conhecer os índices de qualidade nas Unidades Básicas de Saúde e hospitais, e assim, garantir qualidade, segurança e eficácia de um dos principais produtos que auxiliam na manutenção da saúde da população, o medicamento. Isso porque, não somente determina os pontos críticos, mas também au-

\section{REFERÊNCIAS}

1. OMS. Como investigar el uso de medicamentos enlosservicios de salud. Indicadores selecionados del uso de medicamentos. Organização Mundial de Saúde. (DAP. 93.1): OMS, 1993. 87p.

2. Farias AD, Cardoso MAA, Medeiros ACD de, Belém L de $\mathrm{F}$, Simões $\mathrm{M}$ de $\mathrm{O}$ da $\mathrm{S}$. Indicadores de prescrição médica nas unidades básicas de Saúde da Família no município de Campina Grande, PB. Rev. Bras. Epidemiol. 2007. 10 (2): 149- 56.

3. Acúrcio FA. Analysis of Medical Prescriptions Dispensed at Health Centers in Belo Horizonte. Cad. Saúde Pública. xilia na condução de medidas preventivas destinadas a suprimir os erros comprometedores da assistência. Portanto os artigos revisados apontam a necessidade de melhorar a informação dos prescritores sobre o uso racional de medicamentos, visando à redução do numero médio de medicamentos por prescrição.No geral os indicadores de prescrição da OMS são úteis para o conhecimento do perfil da prescrição medicamentosa e comparação com os outros serviços, possibilitando uma possível intervenção, a fim de oferecer uma farmacoterapia eficiente e, consequentemente, a melhoria da qualidade de vida.
2004. 20 (1): 72-79.

4. Cosendey MAE. Análise da implantação do Programa Farmácia Básica: um estudo multicêntrico em cinco estados do Brasil. (Tese). Rio de Janeiro: Escola Nacional de Saúde Pública, Fundação Oswaldo Cruz. 2000.

5. Odubanjo E, Bennett K, Feely J. Influence of Socioeconomic Status on the Quality of Prescribing in the Elderly: a Population Based Study. Br J Clin Pharmacol. 2004. 58 (5): 496-502.

6. Carneiro MFG, Guerra Júnior AFG, Acúrcio FA. Prescrição, dispensação e regulação do consumo de psicotrópicos anorexígenos em Belo Horizonte, Minas Gerais, Bra- 
sil. Cad. Saúde Pública. 2008. 24 (8):1763-1772.

7. Colombo D, Santa H, Ernani T, Agostinho CMG, Didjurgeit JCMA. Padrão de prescrição de medicamentos nas Unidades de Programa de Saúde da Família de Blumenau. Ver. Bras. Ciênc. Farm. 2004. 40 (4).

8. BRASIL. Ministério da Saúde. Agência Nacional de Vigilância Sanitária. Decreto no 3181 de 23 de setembro de 1999. Regulamenta a lei n. 9.797 , de 10 de fevereiro de 1999, que dispõe sobre a vigilância sanitária, estabelece o medicamento genérico, dispõe sobre a utilização de nomes genéricos em produtos farmacêuticos e dá outras providências. Diário Oficial da União, 24 set 1999.

9. Girotto E, Silva PV. A prescrição de medicamentos em um município de Norte do Paraná. Rev. Bras. de Epidemiol. 2006. 9(2): 226-234.

10. Carmo TA, Farhat FCGG, Alves JM. Indicadores de prescrições medicamentosas: ferramentas para intervenção. Saúde em Revista. 2003. 59 (11): 49-56.

11. Cunha MCN, Zorzatto JR, Castro LLC. Avaliação do Uso de Medicamentos na Rede Pública Municipal de Saúde de Campo Grande/MS. Braz. J. Pharm. Sci. 2002. 38 (2): 215-227.

12. Castro CGSO. Estudos de utilização de medicamentos: noções básicas. Rio de Janeiro: Fiocruz, 2002.

13. Dupim JAA, Righi RE. Medicamentos essenciais nos Sistemas Locais de Saúde. In: Bonfim, J.R.A., Mercucci, V.L. A construção da política de medicamentos. São Paulo: Hucitec Sobravime, 1997: 155-163.

14. OMS. Uso racional de los medicamentos. Informe de La Conferencia de Expertos. Nairobi, Genebra; Organização Mundial da Saúde.; 1986.

15. Bermudez AZJ. Indústria Farmacêutica, Estado e Sociedade. São Paulo: Hucitec Sobravime,1995.

16. Romano-Lieber, NS, Teixeira JJV, Goularte FFCL, Ribeiro E, Crozatti MTL Oliveira, GSA. Revisão dos estudos de intervenção do farmacêutico no uso de medicamentos por pacientes idosos. Cad. Saúde Pública. 2002. 18 (6): 1499-1507.

17. Santos V, Nitrini SMOO. Indicadores do uso de medicamentos prescritos e de assistência ao paciente de serviços de saúde. Rev. Saúde Públ. 2004. 38 (6): 819-26.

18. PAHO. Rapid pharmaceutical management assessment: an indicator based approach. Washignton; Pan American Health Organization. 1995.

19. Marcondes NSP. A Assistência farmacêutica básica e o uso de medicamentos na zona urbana do município de Ponta Grossa Paraná: estudo de caso. (Dissertação) Rio de Janeiro: Universidade Estadual de Ponta Grossa/Fun- dação Oswaldo Cruz. 2002.

20. Naves JOS, Silver LD. Evaluation of pharmaceutical assistance in public primary care in Brasilia, Brazil. Rev Saúde Publ. 2005. 39 (2): 223-230.

21. Portela AS, Silva PCD, Simões MOS, Medeiros ACD, Montenegro Neto AN. Indicadores de prescrição e de cuidados ao paciente na atenção básica do município de Esperança, Paraíba. Epidemiologia Serv. Saúde. 2012. 21 (2).

22. Felicio N. Farmacoepidemiologia da prescrição e dispensação de medicamentos na rede pública municipal de saúde de Patos de Minas/MG. (Dissertação) Franca: Universidade de Franca. 2007.

23. Oliveira CAP, Marin MJS, MArchioli M, Pizoletto BHM, Santos RV. Caracterização dos medicamentos prescritos aos idosos na estratégia de saúde da família. Cad. Saúde Públ. 2009. 25 (9).

24. Menezes APS, Domingues MR, Baisch ALM. Compreensão das prescrições pediátricas de antimicrobiano em unidades de saúde em um município do Sul do Brasil. Ver. Bras. Epidemiol. 2009. 12 (3): 478-489.

25. Borges LM; Silva EV. Análises dos indicadores de prescrições médicas em uma unidade da saúde de Anápolis-GO. Rev. Tempus Actas Saúde Colet. 2010: 63-72.

26. Fröhlich SE, Mengue SS. Os indicadores de qualidade da prescrição de medicamentos da Organização Mundial de Saúde ainda são válidos? Rev. Ciênc. e Saúde Coletiva. 2011. 16 (4): 2289-2296.

27. Fleith, VD, Figueiredo MA, Figueiredo KFLRO, Moura EC. Perfil de utilização de medicamentos em usuários da rede básica de saúde de Lorena, SP. Ciênc. Saúde Coletiva. 2008.13 (1): 755-762.

28. Souza JM, Vinholes ER, Trauthman SC, Galato D. Avaliação dos indicadores de prescrição e da demanda atendida de medicamento no sistema único de saúde de um município do sul do estado de Santa Catarina. Rev. de Ciênc. Farm Básica e Apl. 2012. 33 (1): 107113.

29. Zanini AC. Genéricos - o médico decide. Dicionário de medicamentos genéricos. Instituto de defesa do usuário de medicamentos e CRF, DF. São Paulo: IPEX, 1999. p. 4,5.

30. OMS. Guia para a boa prescrição médica. Organização Mundial da Saúde. Porto Alegre: Artmed; 1998. 124p.

31. OMS. Programa de Ação sobre Medicamentos e Vacinas Essenciais. Como estimar as necessidades de Medicamentos. Genebra, Organização Mundial de Saúde. 1989. $102 \mathrm{p}$. 